Wiaczesław Nowikow (D)

Uniwersytet Łódzki wiaczeslaw.nowikow@uni.lodz.pl

\title{
Sobre la subjuntivización diacrónica de la forma castellana en $-r a$
}

\begin{abstract}
Resumen:
En el artículo se examina el problema de la subjuntivización de la forma verbal española cantara que procede del pluscuamperfecto de indicativo latino cantaveram. Actualmente, cantara es alomorfo de cantase siendo el valor gramatical de ambas formas el del imperfecto de subjuntivo. En el trabajo se analiza la alternancia modal en la construcción quiso que cantase / cantara. El material procede del Corpus Diacrónico del Español (CORDE) de la Real Academia Española (RAE) y abarca los años 1580-1930.
\end{abstract}

Palabras clave: modos verbales españoles, indicativo vs. subjuntivo, cantara y cantase, cambio lingüístico, siglos XVI-XX

\begin{abstract}
:
About the Diachronic 'Subjunctivization' of the Castilian -ra Form

The present paper analyses the problem of the 'subjunctivization' of the verbal form cantara, which originates from the Latin pluperfect indicative tense cantaveram. Actually, cantara is the allomorphic form of cantase. Both of the forms have a grammatical value of the imperfect of the subjunctive mood. The modal alternation in the construction quiso que cantase / cantara is discussed. The material used for the purpose of the study comes from the "Corpus Diacrónico del Español" (CORDE) of the Spanish Royal Academy (RAE) and comprises the period from 1580 to 1930 .
\end{abstract}


Keywords: Spanish verbal moods, indicative vs. subjunctive, cantara and cantase, linguistic change, XVI-XX centuries

\section{La evolución y el estatus de cantaveram en las lenguas románicas}

La historia de la forma cantara es una de la particularidades más importantes y uno de los enigmas de la morfosintaxis verbal del español. Varios lingüistas dedicaron numerosos trabajos a la evolución de la forma en -ra que sufrió una transformación modotemporal poco habitual al pasar del pluscuamperfecto de indicativo en latín (cantaveram) al copretérito o imperfecto de indicativo en castellano. Recordemos con este motivo los fundamentales trabajos de L. O. Wright, The -ra verb form in Spain (1932), de A. Veiga, La forma verbal española cantara en su diacronía (1996) y de G. Luquet La teoría de los modos en la descripción del verbo español (2004). El primer estudio incluye el análisis del material procedente de varias obras castellanas y es casi exclusivamente diacrónico, el segundo al tratar las cuestiones teóricas de la descripción del sistema verbal, de hecho, rebasa los límites diacrónicos, mientras que en el tercero, siendo el objetivo fundamental de la monografía la presentación de un nuevo planteamiento en la descripción de los modos verbales, toda la segunda parte (más de la mitad del libro, págs. 89-196) está dedicada al estatus diacrónico-sincrónico de la forma en $-r a$.

Actualmente, la forma cantara se mantiene en castellano, valenciano, gallego y portugués. En dos primeras lenguas, la forma en -ra aparece, básicamente, con valor subjuntivo. Por ejemplo, en valenciano (cfr. Ridruejo, 1985) se dan los empleos en la prótasis de las condicionales no reales:

(1) Si tinguera, daria.

No obstante, cabe recordar que en español existe también la llamada forma indicativa en - $r a$ que al denotar acciones con vector de anterioridad, se da, fundamentalmente, en los lenguajes literario y periódistico: 
(2) Según comunicara el presidente de la nación, las tropas del país vecino habrían cruzado la frontera del Estado hace unas horas.

Un fenómeno semejante existe en el gallego donde al carecer el sistema verbal de las formas compuestas, cantara se emplea, sistemáticamente, con valor del antepretérito (pluscuamperfecto) de indicativo aunque, por influencia del castellano, se dan usos paralelos a la forma en -ra española (p. ej., en las condicionales).

En cambio, en el portugués, salvo el caso de la variedad norteña donde el uso coincide con el del gallego, cantara se emplea con valor del pluscuamperfecto de indicativo propio de tinha cantado en el estilo más bien literario.

Además, conviene notar que actualmente las únicas tres lenguas románicas que continúan el modelo de la prótasis condicional latina $\mathrm{Si}$ habueram son el español, el valenciano y el gallego. Es de suponer que las dos últimas lenguas lo hacen, hasta cierto punto, bajo la influencia del castellano. Recordemos que, como hemos señalado supra, la forma española en -ra, clasificada en el español actual como la de copretérito (imperfecto) de subjuntivo, proviene de la forma del antepretérito (pluscuamperfecto) latino cantaveram.

En resumen, a lo largo de la evolución histórica de varios siglos la forma castellana cantara recorrió el camino desde un indicativo con valor temporal de anterioridad hasta un subjuntivo con vector temporal primario de posterioridad, lo que significa que la forma en - ra fue sometida a dos procesos: al modal de subjuntivización y al temporal de posteriorización.

Dichos procesos fueron iniciados, al parecer, primero en las apódosis y después en las prótasis de las cláusulas condicionales donde cantara venía estableciendo equivalencia funcional con la forma subjuntiva cantase a lo largo de los Siglos de Oro (véase, a este respecto, Mendeloff, 1960; Rojo / Montero Cartelle, 1983; Söhrman, 1991; Nowikow, 1993; Porcar Miralles, 1993; Kítova-Vasíleva, 2018). 


\section{Cantara en los esquemas condicionales y los procesos de subjuntivización y posteriorización (el caso de los siglos XVI-XVII)}

De modo que en el caso de los esquemas condicionales se estaba llevando a cabo, por un lado, el proceso de subjuntivización de cantara que entraba en relaciones de sinonimía gramatical con cantase (los ejemplos de Nowikow, 2011: 207):

(3) Por el alma de mi padre, que ya sé que sois Alijandro, que si fuésedes español, no seríades proveído de melón, sino de buenas razones (Francisco Delicado, "La Lozana Andaluza" [1528], Castalia, Madrid, 1969: 125).

(4) Dezidme ¿si las cartas de que os queremos demandar cuenta fueran latinas, tuviérades por cosa fuera de propósito que os demandáramos cuenta con ellas? (Juan de Valdés, "Diálogo de la lengua" [1535-1540], Cátedra, Madrid, 1984: 121).

En cambio, por el otro, el valor temporal de cantara se venía desplazando desde la zona temporal de anterioridad hacia la de posterioridad (los ejemplos de Nowikow, 2011: 208):

(5) Si ella se hallara presente en aquel debate de la manzana con las tres diosas, nunca sobrenombre de discordis la pusieran (Fernando de Rojas, "La Celestina" [1499], Taurus, Madrid, 1980: 140).

(6) Diana: Luego ¿estáis enamorado de mí?

Carlos: Si no lo estuviera, no me atara este temor.

(Agustín Moreto, “El desdén, con el desdén” [1653-1654], Castalia, Madrid, 1971: 158).

La penetración de la forma en -ra en la prótasis condicional y la expresión temporal primaria de simultaneidad / posterioridad en los esquemas condicionales de los siglos XVI-XVII fueron confirmadas por los resultados del análisis del corpus procedente de los años 1475 - 1662 (véase, a este respecto, Nowikow, 1993, 2011). En total fueron examinadas 47 obras de los años de seis periodos: A. 14751524; B. 1525-1560; C. 1561-1600; D. 1601-1620; E. 1621-1640; 
F.1641-1662. La investigación demostró que en la época áurea el esquema más importante era si tuviera, diera: del total de 2075 ejemplos 782 casos representaron el esquema en cuestión (el 37,2\% de la cantidad global) que, además, predominó en las prótasis con si tuviera (940 ejemplos; el 83,2\%).

Este hecho se ve confirmado por los datos presentados en los cuadros 1 y 2 (Nowikow, 2011: 212-213):

Cuadro 1: Evolución de la "subjuntivización” de si tuviera... entre 1475 у 1662

\begin{tabular}{|c|c|c|c|c|c|c|}
\hline Esquema & $\begin{array}{c}\mathrm{A}(1475- \\
1524)\end{array}$ & $\begin{array}{c}\mathrm{B}(1525- \\
1560)\end{array}$ & $\begin{array}{c}\mathrm{C}(1561- \\
1600)\end{array}$ & $\begin{array}{c}\mathrm{D}(1601- \\
1620)\end{array}$ & $\begin{array}{c}\mathrm{E}(1621- \\
1640)\end{array}$ & $\begin{array}{c}\mathrm{F}(1641- \\
1662)\end{array}$ \\
\hline $\begin{array}{c}\text { Si tuvie- } \\
\text { ra... }\end{array}$ & $24 \%$ & $24 \%$ & $43 \%$ & $57 \%$ & $61 \%$ & $67 \%$ \\
\hline
\end{tabular}

Cuadro 2: Evolución de la "subjuntivización” de si tuviera, diera entre 1475 y 1662

\begin{tabular}{|c|c|c|c|c|c|c|}
\hline Esquema & $\begin{array}{c}\mathrm{A}(1475- \\
1524)\end{array}$ & $\begin{array}{c}\mathrm{B}(1525- \\
1560)\end{array}$ & $\begin{array}{c}\mathrm{C}(1561- \\
1600)\end{array}$ & $\begin{array}{c}\mathrm{D}(1601- \\
1620)\end{array}$ & $\begin{array}{c}\mathrm{E}(1621- \\
1640)\end{array}$ & $\begin{array}{c}\mathrm{F}(1641- \\
1662)\end{array}$ \\
\hline $\begin{array}{c}\text { Si tuvie- } \\
\text { ra, diera }\end{array}$ & $20 \%$ & $19 \%$ & $37 \%$ & $48 \%$ & $52 \%$ & $54 \%$ \\
\hline
\end{tabular}

La conclusión fundamental de estos porcentajes es la siguiente: en los esquemas condicionales el cambio modal, es decir, la subjuntivización de la forma en -ra que se manifestaba a través de la penetración acelerada de cantara en la prótasis condicional, donde esta forma alternaba con el copretérito (imperfecto) de subjuntivo cantase, comenzó aproximadamente a mediados del siglo XVI.

En cambio, la modificación de la reorientación temporal de la forma en -ra realizada mediante el esquema si tuviera, diera, cronológicamente, se presenta de la siguiente manera (I-1 significa irrealidad anterior, mientras que I1+ simboliza irrealidad simultánea / posterior): 
Cuadro 3: Evolución de la reorientación temporal de si tuviera, diera entre 1475 y 1662

\begin{tabular}{|c|c|c|c|c|c|c|}
\hline $\begin{array}{c}\text { Clase } \\
\text { semántica }\end{array}$ & $\begin{array}{c}\mathrm{A}(1475- \\
1524)\end{array}$ & $\begin{array}{c}\mathrm{B}(1525- \\
1560)\end{array}$ & $\begin{array}{c}\mathrm{C}(1561- \\
1600)\end{array}$ & $\begin{array}{c}\mathrm{D}(1601- \\
1620)\end{array}$ & $\begin{array}{c}\mathrm{E}(1621- \\
1640)\end{array}$ & $\begin{array}{c}\mathrm{F}(1641- \\
1662)\end{array}$ \\
\hline $\mathbf{I}-1$ & $64,5 \%$ & $80,3 \%$ & $83,3 \%$ & $51 \%$ & $33 \%$ & $21 \%$ \\
\hline I1+ & $22,6 \%$ & $8,5 \%$ & $9,4 \%$ & $34,6 \%$ & $56,7 \%$ & $64,6 \%$ \\
\hline
\end{tabular}

Los porcentajes permiten concluir que la reorientación temporal ('anterioridad' > 'posterioridad') acelera claramente entre los años 1601 y 1620 (periodo D) y se lleva a cabo definitivamente entre los años 1621 y 1662 (periodos E y F). Esto quiere decir que en la segunda mitad del s.XVII si tuviera, diera entra como un esquema condicional cuyo valor temporal es predominantemente el de posterioridad.

Además, hay una conclusión que se refiere al orden cronológico de los procesos: entre la aceleración de la penetración de cantara en la prótasis (1561) y la aceleración en la reorientación temporal (1601) pasan aproximadamente 40-50 años.

No obstante, en las cláusulas condicionales, en primer lugar en la apódosis, pero también en la prótasis, la forma en -ra podía alternar no solo con la forma subjuntiva cantase, sino también con los tiempos del modo indicativo tales como, por ejemplo, cantaba y cantaría. Con este motivo, cabe recordar que en español, a nivel gramatical, la modalización proposicional puede llevarse a cabo de dos maneras: mediante el modo subjuntivo y a través del desplazamiento temporal de los tiempos del modo indicativo. Este segundo mecanismo se da a menudo en las oraciones condicionales a través del empleo de formas tales como cantaba y cantaría que alternan en la apódosis (la última es más habitual, pero la primera también es posible). En algunas lenguas, como en francés, provenzal o catalán, el uso del indicativo es canónico dándose el imperfecto de indicativo regularmente en la prótasis: fr. si j'avais, je donnerais; prov. s'aviá, donariá; cat. si tenia, daria, lo que, de hecho, ocurría ya en el latín.

Todo esto significa que en el caso de los esquemas condicionales no siempre tenemos que ver con el proceso de subjuntivización de cantara, forma que, recordemos, procedía del pluscuamperfecto de 
indicativo latino que aparecía en el esquema condicional si habueram, dederam ya en el latín. Es de subrayar que al desplazarse temporalmente, la forma en - ra denotaba anterioridad ya no solo con respecto a un momento anterior al origen (expresado este, p. ej., por lat. dixi / esp. dije), valor temporal propio del pluscuamperfecto, sino con referencia al propio origen (marcado este, p. ej., por lat. dico / esp. digo).

Estas observaciones nos llevan a la conclusión de que el llamado periodo condicional no es el entorno sintáctico-semántico más idóneo para determinar en qué fase se produce el cambio del estatus gramatical de la forma en -ra. En nuestra opinión, el criterio de la subjuntivización de cantara debería ser establecido a partir de la distribución de contraste modal máximo donde el empleo del modo indicativo es imposible.

\section{La subjuntivización a través de las cláusulas subordinadas sustantivas (¿el siglo XVII?)}

El contraste de este tipo se da en el caso de la selección modal en las cláusulas subordinadas sustantivas (nominales) después de los predicados de voluntad (deseo, necesidad; querer, desear, apetecer, ser necesario, etc.) que, al lado de los de influencia (mandato, ruego, prohibición, permiso; ordenar, pedir, prohibir, permitir, etc.) y causatividad (causar, originar, provocar, etc.), forman parte de la distribución con el subjuntivo obligado.

Como es sabido, en castellano después de querer o ser necesario siendo el indicativo imposible, el empleo del subjuntivo es obligatorio. Parece ser un hecho universal relacionado con la semántica de los predicados en cuestión, puesto que estos últimos se combinan en algunas lenguas con el subjuntivo (lenguas románicas) y, en otras, con el modo hipotético (lenguas eslavas). Como sea, en ambos casos el modo seleccionado no es el indicativo (véase, a este respecto, Nowikow, 2017: 69-71).

Con respecto a la fecha de la subjuntivización de cantara, se suele señalar el siglo XVII como el periodo del comienzo de la consolidación de esta forma en el modo subjuntivo. No obstante, esta tesis, 
que sepamos, no ha sido confirmada mediante los datos procedentes de una investigación basada en el examen de un corpus diacrónico amplio y representativo, salvo el caso de los esquemas condicionales comentado supra. Por otro lado, es verdad que las observaciones sobre la importancia del siglo XVII para la evolución modal de la forma en -ra aparecen en varios estudios, entre otros, en los de Luquet (1988), Cano Aguilar (1990), Cabeza (1990), Nowikow (1993), Veiga (1996), Kítova-Vasileva (2018).

No faltan ejemplos del empleo de cantara con valor gramatical del subjuntivo en las cláusulas subordinadas nominales después de los predicados de voluntad y/o influencia:

(7) esto le dije yo que advirtiera que en la Corte había de todo (Buscón, 169) [Cabeza, 1990: 327].

(8) Díjome que le siguiera a su casa, y [...] llegamos con brevedad a ella (Estebanillo González, 249) [Nowikow, 1993: 177].

Sin embargo, incluso para los siglos XVI-XVII (y mucho menos para los posteriores), a falta de un estudio más sistemático, se trata de ejemplos, de hecho, aislados y no muy regulares.

\section{La subjuntivización de cantara en la construcción con quiso que entre 1530 y 1930 (la frecuencia absoluta y los porcentajes)}

Por lo tanto, hemos decidido realizar una pequeña investigación de carácter introductorio para comprobar la evolución del empleo de cantara como exponente del modo subjuntivo en las cláusulas subordinadas sustantivas regidas por el núcleo predicativo (NP1) con el verbo querer en forma de pretérito: quiso que +NP2. Hemos centrado nuestra atención en la alternancia de las formas cantase y cantara al estudiar los casos que corresponden al esquema sintáctico

NP1 (subordinante) quiso + que + NP2 (subordinado) cantase / cantara / X.

Es evidente que se trata de uno de los esquemas más frecuentes con respecto a los predicados de voluntad e influencia. El símbolo X 
se refiere a otras formas (p. ej., cante) que aparecieron, sin embargo, en muy pocas ocasiones.

El material analizado proviene del Corpus Diacrónico del Español (CORDE) de la Real Academia Española (REAL ACADEMIA ESPAÑOLA: Banco de datos (CORDE) [en línea]. Corpus diacrónico del español. http://www.rae.es, 18.04.2019 y 29.04.2019]). Hemos distinguido siete periodos cronológicos de 50 años de duración cada uno: 1580-1630 (577 casos en 179 documentos: 577 / 179), 1631-1680 (137 / 57), 1681-1730 (46 / 12), 1731- 1780 (39 / 26), 1781 - 1830 (76 / 44), 1831 - 1880 (139 / 94), 1881 - 1930 (205 / 109). Evidentemente, la división cronológica es arbitraria siendo su objetivo principal poner de relieve las líneas evolutivas de cantara a lo largo de 350 años. En total, el corpus incluye 1219 formas en -se y -ra registradas en 521 documentos. En cambio, la selección del predicado subordinante (NP1) quiso no fue arbitraria. Según la lista de frecuencias del CORDE, es una de las formas verbales personales más frecuentes (frecuencia absoluta: 39594; frecuencia normalizada: 169.427). Se encuentra en el lugar 485 de la lista de frecuencias de 1000 elementos léxicos y gramaticales más usados. Además, es la forma personal de pretérito más frecuente adelantada en la lista del CORDE solo por la forma personal de presente quiero. En general, el verbo querer se encuentra entre los 13 verbos más usados al lado de ser, estar, tener, haber, decir, hacer, poder, ir, poner, saber y llegar siendo el más frecuente entre los predicados de influencia.

A continuación, en el cuadro 3, presentamos los resultados de nuestro análisis de la evolución cuantitativa del empleo de cantase y cantara en los textos procedentes de los años 1580-1930:

Cuadro 4: Evolución de cantara (vs. cantase) en la construcción "quiso que + NP2" entre 1580 y 1930

\begin{tabular}{|c|c|c|c|}
\hline Periodo & $\begin{array}{c}\text {-se + -ra: frecuencia } \\
\text { absoluta (cantidad de } \\
\text { documentos) }\end{array}$ & $\begin{array}{c}\text { la forma en -ra: } \\
\text { frecuencia abso- } \\
\text { luta }\end{array}$ & $\begin{array}{c}\text { la forma en -ra: } \\
\text { porcentaje }\end{array}$ \\
\hline $1580-1630$ & $577(179)$ & 8 & $1,38 \%$ \\
\hline
\end{tabular}




\begin{tabular}{|c|c|c|c|}
\hline $1631-1680$ & $137(57)$ & 6 & $4,37 \%$ \\
\hline $1681-1730$ & $46(12)$ & 20 & $43,47 \%$ \\
\hline $1731-1780$ & $39(26)$ & 3 & $7,68 \%$ \\
\hline $1781-1830$ & $76(44)$ & 13 & $17,1 \%$ \\
\hline $1831-1880$ & $139(94)$ & 47 & $33,81 \%$ \\
\hline $1881-1930$ & $205(109)$ & 97 & $47,31 \%$ \\
\hline TOTAL & $1219(521)$ & 194 & \\
\hline
\end{tabular}

Como se ve, operamos, básicamente, con el parámetro de frecuencia general o absoluta que se refiere al número de veces que determinada forma gramatical aparece en el total del corpus. No hemos recurrido en este caso al parámetro de frecuencia normalizada que permite comparar un fenómeno (o una forma) en corpus de diferentes tamaños. La frecuencia normalizada se refiere al número de veces que aparece una determinada forma por cada millón de elementos o a la expresión porcentual sobre el total de formas del corpus (p. ej., 30 688, 85 vs. 3,07\%). En cambio, hemos señalado la cantidad de documentos examinados en cada uno de los periodos. Sin entrar en los detalles, cabe subrayar que, grosso modo, existe cierta dependencia entre las frecuencia absoluta y normalizada: mayor cantidad de formas suele estar relacionada con mayor número de textos. Por otro lado, se mantienen ciertas proporciones entre formas y documentos: por ejemplo, si la cantidad de documentos de un periodo es dos o tres veces más grande que la de otra etapa cronológica, la diferencia entre las cantidades de formas registradas en las dos fases mantiene más o menos las mismas proporciones. De todos modos, el factor de frecuencia normalizada habría sido más importante si hubiésemos comparado diferentes variedades diatópicas, diastráticas o diafásicas, lo que no es objeto de este estudio.

\section{La evolución entre 1580 y 1680}

Con respecto al siglo XVII, los resultados parecen ser, hasta cierto punto, bastante contradictorios. En los periodos de 1580-1630 y 16311680 los porcentajes del llamado subjuntivo no irreal empleado fuera 
de las oraciones condicionales (en este caso en construcción con quiso que) son muy bajos (1,38\% en el primero y 4,37\% en el segundo) y confirman las observaciones de Cabeza (8 casos sobre 274 apariciones de cantara; 1990: 327): "los usos no irreales de cantara no pasan de ser meros atisbos que muestran el inicio de una nueva tendencia de la lengua” (1990: 328). Por otro lado, los casos registrados, en su mayoría, no suscitan dudas y demuestran los primeros pasos de cantara hacia el paralelismo distribucional y modal con cantase, lo que se comprueba, por ejemplo, a través del siguiente fragmento:

(9) Estas sediciones se iban cada día encendiendo más con la ocasión de estarse ociosos en el pueblo, por la que daban las aguas a no poder salir, cuando un alguacil del campo, habiéndole quitado su caballo por haberse ejecutado en él el primero el decreto que el gobernador había mandado publicar, habiéndolo quebrantado, quiso que también se ejecutara en otro que habia contravenido también a lo mismo (1612-1625, Márquez, Fray Juan, El gobernador cristiano, numeración en el CORDE: párrafo 445, páginas I, 311).

Además, hemos documentado, entre otros, usos tales como

(10) ...no quiso que un cuarto fuera (1627, Simón, Fray Pedro, Primera parte de noticias historiales de las conquistas de tierra firme en las Indias Occidentales, párrafo 521).

(11) ...permitir no quiso que muriera (1597-1645, Quevedo y Villegas, Francisco de, Poesías, párrafo 549).

(12) Para nombrallos quiso que salieran (1627, Carvajal y Robles, Rodrigo, Poema del asalto y conquista de Antequera, párrafo 554).

(13) ... y quiso que descansara en tu casa (1620-1623, Ruiz de Alarcón, Juan, El dueño de las estrellas, párrafo 573).

Dichas secuencias confirman que, al parecer, no había restricciones léxicas en cuanto a las combinaciones de quiso que con diferentes verbos, lo que, en principio, debía favorecer la difusión del paralelismo modal de cantara con cantase.

La situación parece cambiar radicalmente en el periodo 1681-1730. Sin embargo, el aumento de la frecuencia absoluta es, hasta cierto 
punto, engañoso. De 20 casos de cantara en construcción con quiso que 17 provienen de la obra "El sabio instruido de la Gracia” (1703) de Francisco de Garau. Además, este periodo se caracteriza por la menor cantidad de documentos (12). Todo esto quiere decir que en el resto de 11 documentos solo se han registrado 3 formas en -ra empleadas después de quiso que. De modo que a lo mejor tenemos que ver con un hecho estilístico: el uso preferido de un autor en una de sus obras.

\section{Observaciones finales}

El crecimiento más significativo del empleo de cantara en construcción con quiso que parece empezar en el periodo 1781-1830 cuando el porcentaje al llegar a $17,1 \%$, por primera vez se acerca a un $20 \%$. La consolidación del fénomeno sigue y aumenta en los periodos de 1831-1880 y 1881-1930 cuando el porcentaje de quiso que cantara alcanza el 33,81\% y el 47,31\%, respectivamente. Si comparamos estos porcentajes con los de dos primeros periodos (1,38\% y 4,37\%), vemos que a lo largo de trescientos años se produjo un cambio sustancial en la característica modal de cantara.

En resumen, en el caso que hemos examinado, la subjuntivización de cantara a través de las subordinadas nominales regidas por el núcleo predicativo quiso que parece acelerar a partir de finales del siglo XVIII (1781) consolidándose definitivamente hacia finales del XIX y principios del XX. Entre 1730 y 1930 el porcentaje de cantara subjuntivo sube de 17,1 \% a 47, $31 \%$, es decir, aumenta en más de un $30 \%$. En el periodo de 1881-1930 la frecuencia absoluta de cantara casi equivale a la de cantase ( 97 formas en -ra frente a 109 formas en -se). La conclusión final es la siguiente: en el caso de la construcción quiso que cantara no parece que haya motivos para hablar sobre la especial importancia del castellano del siglo XVII para la subjuntivización de la forma en -ra. Los cambios decisivos para el estatus gramatical de cantara, es decir, para su subjuntivización, empiezan entre cien y doscientos años más tarde. 


\section{Bibliografía}

CABEZA, C. (1990), "Valores de la forma 'cantara’ en el español clásico”, en: Actas del II Congreso Internacional de Historia de la Lengua Española, vol. I, Pabellón de España, Madrid, pp. 323-331.

CANO AGUILAR, R. (1990), "Sobre la historia del subjuntivo español", en: Actas del Congreso de la Sociedad Española de Lingüística. XX Aniversario, vol. I, Gredos, Madrid, pp. 340-353.

KÍTOVA-VASÍLEVA, M. (2018), Los esquemas condicionales en el Diálogo de la lengua de Juan de Valdés, Colección Yuso, vol. 10, Editorial Axac, Lugo, https://doi.org/10.3989/rfe.2019.012.

LUQUET, G. (1988), Systématique historique du mode subjonctif espagnol, Klinksieck, Paris.

LUQUET, G. (2004), La teoría de los modos en la descripción del verbo español, Arco / Libros, S. L., Madrid.

MENDELOFF, H. (1960), The Evolution of the Conditional Sentence Contrary to Fact in Old Spanish, The Catholic University of America Press, Washington.

NOWIKOW, W. (1993), Evolución funcional de los esquemas condicionales no reales en el español de los Siglos de Oro, Wydawnictwo Uniwersytetu Łódzkiego / Vervuert Verlag, Łódź-Frankfurt.

NOWIKOW, W. (2011), "Sobre el esquema condicional más plurifuncional en la historia del castellano: en torno a la interpretación del cambio de si tuviera, diera en los siglos XVI-XVII”, en: Sinner, C., Ramírez Luen-

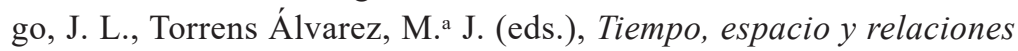
espacio-temporales desde la perspectiva de la lingüistica histórica, Cilengua, San Millán de la Cogolla, pp. 205-226.

NOWIKOW, W. (2017), "Sobre la bifuncionalidad del modo Subjuntivo: ¿función sintáctica o / y función semántica?”, Moenia, 23, pp. 61-72.

PORCAR MIRALLES, M. (1993), La oración condicional: la evolución de los esquemas verbales condicionales desde el latín al español actual, Universitat Jaume I, Castelló.

RIDRUEJO, E. (1985), “La forma verbal en - ra en valenciano”, en: Actes du XIIème Congrès International de Linguistique et Philologie Romanes, 2, Université de Provence, Aix-en-Provence, pp. 437-448. 